\title{
Review \\ Clinical trials in systemic sclerosis: lessons learned and outcomes
} Marco Matucci-Cerinic ${ }^{1}$, Virginia D Steen ${ }^{2}$, Daniel E Furst ${ }^{3}$ and James R Seibold ${ }^{4}$

\author{
1Division of Medicine and Surgery, Division of Medicine I and Rheumatology, Villa Monna Tessa, Viale Pieraccini, I-50139 Florence, Italy \\ 2Division of Rheumatology, Immunology and Allergy, Georgetown University Hospital, Reservoir Road NW, Washington, District of Columbia 20007, USA \\ ${ }^{3}$ David Geffen School of Medicine at UCLA, Veteran Ave, Los Angeles, California 90085-1670, USA \\ 4 University of Michigan Scleroderma Program, East Medical Center Drive, Ann Arbor, Michigan 48109-0358, USA
}

Corresponding author: Marco Matucci-Cerinic, cerinic@unifi.it

Published: 15 August 2007

This article is online at http://arthritis-research.com/content/9/S2/S7

(c) 2007 BioMed Central Ltd
Arthritis Research \& Therapy 2007, 9(Suppl 2):S7 (doi:10.1186/ar2191)

\section{Lessons from clinical trials in systemic sclerosis}

Disease modification in SSc means extension of lifespan. With this in mind, several drugs are now available to manage the specific manifestations of scleroderma, including angiotensin-converting enzyme inhibitors for renal crisis [1] and epoprostenol for pulmonary arterial hypertension (PAH) [2]. Available data for the dual endothelin receptor antagonist bosentan suggest that it may also be considered a life-prolonging agent in the setting of PAH-SSc [3], and it is hoped that cyclophosphamide will be as effective in pulmonary fibrosis (PF) [4]. However, although significant advances are being made, these are mainly with respect to specific organ involvement. In the following sections some of these recent clinical trial data are reviewed to show how they are contributing to our understanding of therapeutic approaches that may be appropriate in the management of SSc. We also highlight key observations that have been reported and are helping to shape the future of clinical trials.

Introduction
As is comprehensively reported in the previous reviews in this supplement, systemic sclerosis (SSc) is a multisystem disorder with a complex pathogenesis that remains to be elucidated. It is apparent that a combination of environmental or genetic factors leads to early immune activation and even earlier vascular damage, which leads ultimately, through a variety of signals, to myofibroblast differentiation and proliferation. The net result of these perturbations is profound vascular damage and tissue injury, characterized by fibrosis and remodelling. This review examines data from clinical trials as well as those from observational databases and registries. The aim is to present information gleaned from recent clinical experience of current therapeutic approaches and to highlight the many challenges to the SSc community that still remain to be overcome.

\section{Nonspecific antifibrotic agents \\ $D$-penicillamine and relaxin}

The anticollagen and immune-modulating effects of Dpenicillamine provided a rationale for testing its efficacy in diffuse cutaneous SSc, specifically to slow skin fibrosis. However, although some observational studies initially appeared to demonstrate improved skin thickening, significant benefit in 5-year mortality and a decrease in development of visceral disease, these benefits were not confirmed in a well controlled, randomized, double-blind trial [5]. This trial enrolled 134 patients who were approximately 10 months into their disease and had a mean skin score of about 20. After 24 months, no significant differences in skin

$\mathrm{DLCO}=$ carbon dioxide diffusing capacity; $\mathrm{DU}=$ digital ulcer; $\mathrm{ET}=$ endothelin; FVC = forced vital capacity; HAQ = Health Assessment Questionnaire; ILD = interstitial lung disease; mRss = Modified Rodnan Skin Score; $6 \mathrm{MWD}=6$ min walk distance; PAH = pulmonary arterial hypertension; $\mathrm{PF}=$ pulmonary fibrosis; SSc $=$ systemic sclerosis; UCLA = University of California, Los Angeles. 
scores were observed between patients receiving low doses of D-penicillamine (125 mg every other day) and those receiving usual doses (750 to $1,000 \mathrm{mg} /$ day).

Relaxin is an antifibrotic pregnancy-related hormone that is responsible for loosening the pelvic ligaments to facilitate parturition [6]. At the transcriptional level, relaxin reduces the production of collagen types I and III, enhances collagenase types I and III, and reduces the level of tissue inhibitor of matrix metalloproteinase. Thus, relaxin has three putative antifibrotic mechanisms. A phase II study comparing relaxin with placebo in patients with diffuse SSc in later stages yielded promising results [7]. In the $25 \mu \mathrm{g}$ patient cohort, a statistically significant reduction in skin score from 27 to 19 by week 24 was observed. Disappointingly, these results were not repeated in a subsequent phase III study involving 231 patients, despite entry skin scores and other characteristics being identical to those in the phase II study (Seibold JR, unpublished data). This study, which compared the effective dose from the phase II study $(25 \mu \mathrm{g})$ with a $10 \mu \mathrm{g}$ dose and placebo, detected identical responses for the $25 \mu \mathrm{g}$ dose and placebo.

\section{Methotrexate}

Two randomized, double-blind, placebo-controlled trials evaluated the antifolate drug methotrexate in patients with SSc $[8,9]$. In the first of these trials, 29 SSc patients $(37.9 \%$ with diffuse disease; mean disease duration about 3 years) were randomly assigned to receive methotrexate $15 \mathrm{mg} /$ week for 24 weeks [8]. After the initial 24-week double-blind period, patients who responded favourably (improvement in total skin score, visual-analogue scale improvement of $\geq 30 \%$, or improvement in carbon dioxide diffusing capacity [DLCo] of $\geq 15 \%$ ) continued for a further 24 weeks on the same treatment in an observational extension phase. Those who responded poorly on placebo were given methotrexate $15 \mathrm{mg} /$ week and those who responded poorly on methotrexate had their dose increased to $25 \mathrm{mg} /$ week. Although the patient numbers were small, significantly more patients on methotrexate responded favourably compared with the placebo group $(P=0.03)$. However, improvement in individual measures only tended toward significance. Of the 22 patients who received methotrexate for at least 24 weeks, and completed the trial, $68 \%$ responded to methotrexate therapy.

A more recent randomized, double-blind trial was conducted in 71 early diffuse SSc patients (duration < 3 years; mean duration $<7.5$ months), who were randomly assigned to receive either methotrexate $(n=35)$ titrated up to $15 \mathrm{mg} /$ week or placebo $(n=36)$ for 12 months [9]. Baseline skin scores were similar in the two groups; the mean University of California, Los Angeles (UCLA) score was about 11 and the modified Rodnan skin score (mRss) was about 27. Although the study concluded that there was little evidence that methotrexate is significantly effective in the treatment of early diffuse SSc, there were some significant differences between patients given methotrexate and those who received placebo. In patients who completed the trial $(n=54)$, UCLA skin scores were significantly better in the methotrexate group than in the placebo group after 3 months $(P<0.05)$, although this difference was not maintained at 12 months. The mRss improved over the course of the study, and although differences compared with placebo never reached formal significance, such a trend was present and was supported by the UCLA score. At 12 months, despite the lack of quantitative differences in skin scores and other parameters, such as patient global assessment, Health Assessment Questionnaire (HAQ) pain and disability, and grip strength, the physician global assessment was significantly better for patients given methotrexate $(P<0.035)$. Overall, this study supported the initial study of van den Hoogen and coworkers [8], although it was far from a ringing endorsement.

\section{Summary}

Some patients appear to benefit from methotrexate, although a substantial proportion fails to respond to therapy. The disappointing results with both $\mathrm{D}$-penicillamine and relaxin may be because these agents are not well suited to SSc pathophysiology as both can be considered nonspecific antifibrotic agents. However, a valuable lesson from these trials was the finding of a quantifiable, spontaneous improvement in some patients with SSc.

\section{Targeting the systemic sclerosis-specific pathophysiology}

The largely disappointing results from the trials of nonspecific antifibrotic agents prompted an evaluation of cumulative data on ineffective drugs and placebo arms of trials, which was performed by a core panel of trained experts [10]. Forced vital capacity (FVC), mRss, and HAQ findings from 645 patients with early diffuse SSc were evaluated to gain information on disease progression in these patients. The results indicated that although patients entered the studies with an mRSS in the region of 27 , this reduced to about 13 at the 24-month point, representing a 50\% spontaneous reduction over 2 years. Interestingly, patients who entered trials with relatively mild disease at the outset tended to worsen, and those that entered with relatively severe disease tended to exhibit spontaneous improvement. This demonstrates significant regression to the mean, and indicates that establishment of stable baseline data and good training are necessary to decrease variability of measurements so that realistic targets for analysis can be set.

The observations of spontaneous improvement of skin in patients with diffuse SSc led to the investigation of disease progression in the lung. In contrast to idiopathic pulmonary fibrosis, an aggressive and rapidly progressive condition, interstitial lung disease (ILD) secondary to SSc (SSc-ILD) progresses more slowly and is well recognized for its stability after an initial 1-year or 2-year period of decline [11]. The cumulative analysis of the clinical trial data [10] showed that 
patients with early diffuse SSc entered and left the trials with $\%$ FVC values in the high 70s. Thus, ILD tends to accrue early but is more stable in later stages. It may therefore be that it is not progressive interstitial fibrosis but rather progressive vascular injury in the lung that leads to a diminishing diffusing capacity over time. Surprisingly, however, diffusing capacity (\%DLCo) at entry was in the low 60 s and tended to remain stable.

Some positive results with methotrexate have been observed in relatively small numbers of SSc patients. However, the combined findings of these trials and of the evaluation of ineffective nonspecific therapies and placebo data suggest that a more specific approach, through targeting perturbations that are typical of SSc, may be more appropriate and may yield more promising results.

\section{CAT-192}

As presented in other reviews in this supplement [12,13], transforming growth factor- $\beta$ appears to be an important mediator of much of the pathophysiology of SSc, particularly in terms of extracellular matrix deposition and vasculature injury. A recent study of CAT-192, which is a fully humanized, monoclonal antibody to transforming growth factor- $\beta_{1}$, examined change in mRss over 6 months in patients with diffuse, progressive SSc in its earliest possible stage, who had a median disease duration at baseline of just 6.4 months [14]. Disappointingly, there was no good evidence to suggest that the drug had any effect on mRss, but this findings could have been a reflection of regression toward the mean or the very small number of patients in each group ( $n=7$ to 9 ), or it could have been due to the lack of robust metrics available to measure the natural history of the disease. The lesson from this study is that adequate power is critical, even in early phase I studies, if treatment effects are to be demonstrated in subsequent studies.

\section{Bosentan: BUILD-2 trial}

Pulmonary complications, including interstitial fibrosis and pulmonary vasculopathy, are now the leading causes of death in patients with SSc. There is considerable evidence to suggest that endothelin (ET)-1 is a key participant in many of the pathological features of SSc. Plasma levels are elevated in SSc patients, particularly those with PAH or ILD, and ET-1 is over-expressed in as-yet-uninvolved and early-involved skin [15]. Furthermore, SSc lung fibrosis is associated with elevated ET-1 ligand expression and increased expression of endothelin receptor subtype $B$ binding sites. Bosentan, a dual endothelin receptor antagonist, has been shown to improve exercise capacity, haemodynamic parameters, time to clinical worsening and quality of life in patients with PAH related to connective tissue disease, including SSc [16-18].

The BUILD-2 (Bosentan in Interstitial Lung Disease in Systemic Sclerosis-2) trial [19] compared the effects of bosentan with those of placebo on clinical outcomes of
Figure 1

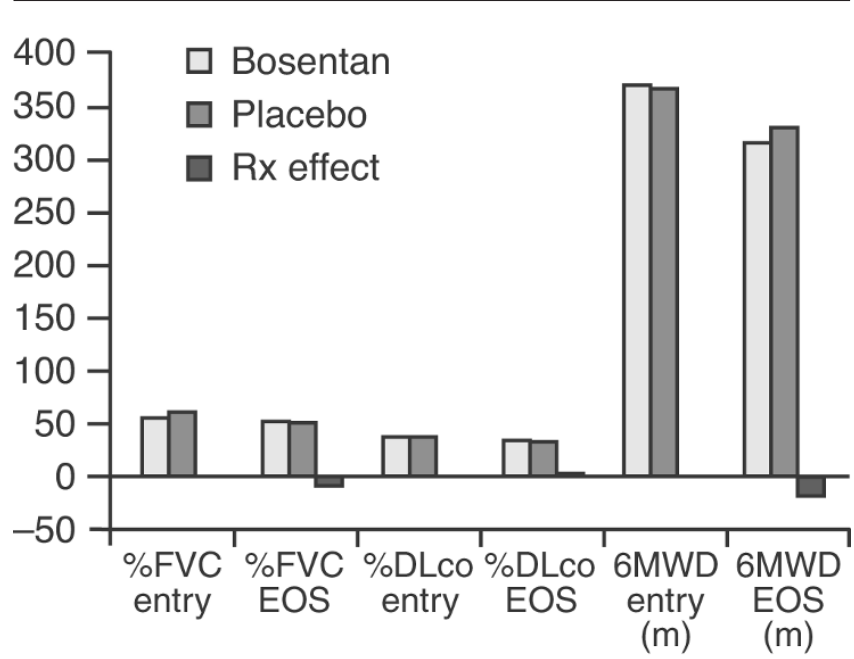

Key outcomes for the BUILD-2 study of bosentan versus placebo in SSc-ILD. BUILD, Bosentan in Interstitial Lung Disease in Systemic Sclerosis; DLCO, carbon dioxide diffusing capacity; EOS, end of study; FVC, forced vital capacity; ILD, interstitial lung disease; 6MWD, 6 min walk distance; Rx, treatment; SSc, systemic sclerosis.

patients with SSc with ILD. It was designed to select patients with progressive disease. The primary outcome measure was 6-minute walk distance (6MWD) at 12 months; secondary measures included dyspnoea indices, pulmonary function and other SSc features (including mRss and scleroderma HAQ), and quality of life (QoL).

The study found no differences in effect on 6MWD and no effect on key secondary outcomes, including FVC and DLCO, and time to desaturation (Figure 1) [19]. This might have been due to the 6MWD not being appropriate for assessing treatment effects in parenchymal lung disease or to the fact that, despite efforts to select a population with progressive disease, the patients enrolled in the study had relatively stable disease. Progression of SSc is characterized by periods of spontaneous improvement in skin scores and stabilization of pulmonary function tests, which confounds the selection of patients with progressive disease. Although this trial did not succeed in demonstrating that bosentan improved or stabilized SSc-ILD, it did initiate debate on validation of the 6MWD test in SSc and suggested that there is a need for measures that are sufficiently sensitive to changes throughout the duration of the study [20].

\section{Bosentan: RAPIDS-1 and RAPIDS-2 (bosentan in digital ulcers)}

Given the potentially critical role of ET-1 in the pathophysiology of SSc, additional areas of interest are other vascular manifestions of the disease. Digital ulcers (DUs) are a major cause of morbidity in SSc patients, with at least $50 \%$ of patients experiencing DUs at some point in their disease course. DUs represent a significant burden of the disease. 
They are excruciating, unsightly and frequently become infected. Those that become gangrenous or a nidus of deeper infection frequently autoamputate or require formal surgical amputation.

RAPIDS-1 (RAndomized Placebo-controlled Investigation of Digital ulcers in Scleroderma) demonstrated that bosentan could reduce the number of new DUs [21]. In this randomized, prospective, placebo-controlled, double-blind study, 122 patients with a history of a documented DU within the previous 12 months were randomly assigned to drug (bosentan $125 \mathrm{mg}$ twice daily) or placebo. After 12 weeks, although bosentan therapy had no effect on the healing of existing DUs, the mean number of new DUs during this period was 2.7 in patients given placebo and 1.4 in those who received bosentan, representing a reduction of $48 \%$. The lack of a reported effect on healing might have been partly due to difficulties in the precise identification and measurement of DUs. Photographing DUs is not a viable approach because of technical issues, and no fully validated measurement for DUs has yet been developed.

A further trial, RAPIDS-2 [22], built upon the findings of RAPIDS-1 and examined the effects of bosentan treatment on a designated cardinal ulcer. The results were in agreement with those from the RAPIDS-1 study, and the consistency of findings between these two large, placebo-controlled trials supports the use of bosentan to reduce the number of new DUs in SSc and a role for endothelin in maintaining vascular integrity and function. However, bosentan was not associated with improved healing of existing active ulcers.

An important lesson from these studies is that validated measurement techniques must be defined and clinical trials sufficiently powered if they are to detect differences in outcome in SSc.

\section{Cyclophosphamide in interstitial lung disease}

A double-blind, randomized, placebo-controlled trial of the oral immunosuppressant cyclophosphamide was conducted in 158 patients with SSc with evidence of ILD by highresolution computed tomography or bronchoalveolar lavage [4]. The enrolled patients received treatment with the oral immunosuppressant cyclophosphamide ( 1.5 to $2 \mathrm{mg} / \mathrm{kg}$ per day) or matching placebo for 1 year, and were followed for an additional year. Of 158 patients, 145 completed at least 6 months of treatment and were included in the analysis. The $\%$ FVC predicted stabilized or improved in patients on cyclophosphamide and worsened in patients on placebo. There was a mean absolute difference in adjusted 12-month $\%$ FVC predicted between the cyclophosphamide and placebo groups of $2.53 \%$, favouring cyclophosphamide $(P<0.03$; Figure 2). When this effect was adjusted for the level of fibrosis, the effect increased to approximately $2.9 \%$. A year of cyclophosphamide therapy for a 3\% change in FVC is, at face value, somewhat difficult to accept. Nevertheless,
Figure 2

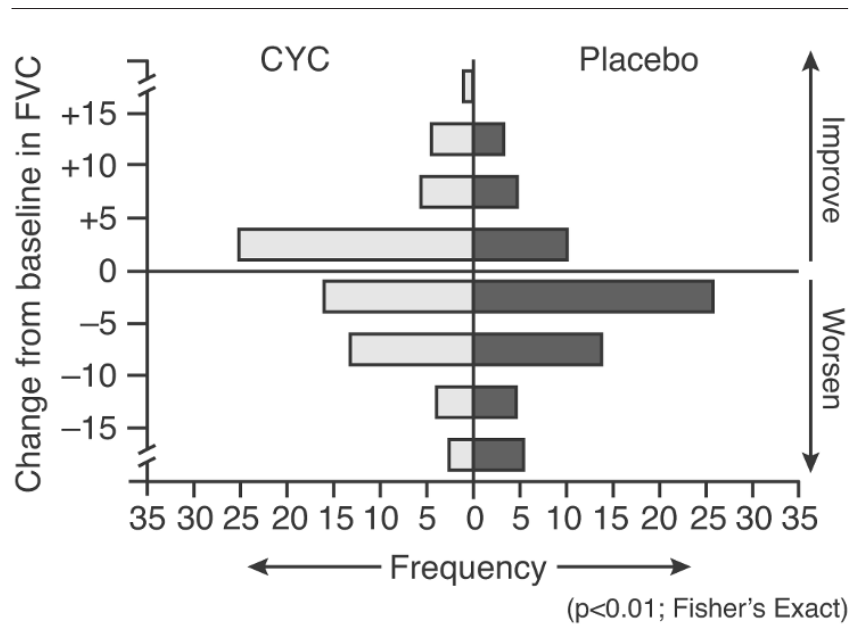

Cyclophosphamide versus placebo: effect on FVC. CYC, cyclophosphamide; FVC, forced vital capacity. Reproduced with permission from Tashkin et al. N Engl J Med 2006 Copyright (C) 2006 Massachusetts Medical Society [4].

there was evidence of benefit in this trial, particularly in patients with more severe disease. This confounds the prevention paradigm in ILD, but it makes an argument that some of this effect may be driven by severity. A change of 1 point in the transitional dyspnoea index focal score is considered clinically meaningful [23], and there was a significant improvement in the cyclophosphamide group compared with placebo (+1.4 versus $-1.3 ; P<0.001)$. Changes favouring cyclophosphamide were also seen for skin score, the scleroderma HAQ, the Transitional Dyspnoea Index and components of the 36-item Short Form, which indicates that individuals with SSc and ILD benefited in more than one way from cyclophosphamide therapy. Further analysis will be needed to determine whether this study can be instructive regarding which patients are most likely to benefit from treatment. Neither bronchoalveolar lavage nor unvalidated, semiquantitative scoring of high resolution computed tomography data were predictive of clinical course or response to therapy.

It appears that careful patient selection, adequate numbers, good quality assurance and sophisticated analysis are all necessary when testing modestly effective drugs in SSc.

\section{Autologous stem cell therapy}

An open-label pilot study of immunoablation followed by stem cell replacement demonstrated dramatic improvements in outcome in 33 SSc patients with primary organ involvement. At baseline, patients had high mRss (median 30), a moderately decreased DLCo (median 62\%), mildly decreased FVC (median 72\%) and relatively early disease (median duration 20.5 months). The mRss decreased to approximately 5 after 4 years (Figure 3). There was also a dramatic 
Figure 3

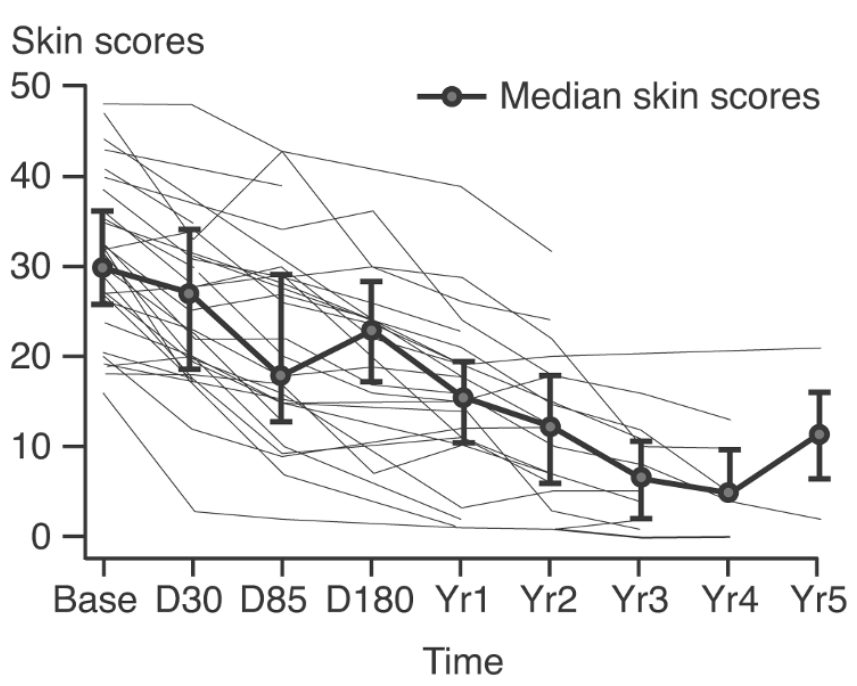

Pilot study showing mRss over time following immunoablation therapy. mRss, Modified Rodnan Skin Score. This research was originally published in Blood Online. Nash RA et al. High-dose

immunosuppressive therapy and autologous hematopoietic cell transplantation for severe systemic sclerosis: long-term follow-up of the U.S. multicenter pilot study. Blood. Prepublished online April 23, 2007; DOI 10.1182/blood-2007-02-072389 [24].

improvement in $\mathrm{HAQ}$ to almost zero in terms of the group mean. Visceral involvement stabilized [24]. Although this was an open study and subject to all of the significant problems of such trials, the apparent dramatic responses are encouraging and indicate that the measurements we use at present may be adequate for highly effective therapeutic options. A multicentre, randomized, controlled, National Institutes of Health approved trial of these approaches has started in the USA.

The European Group for Blood and Marrow Transplantation/ European League Against Rheumatism reported an analysis of their database of 57 patients with SSc treated by haematopoietic stem cell transplantation, with more than 6 months of follow up, in European phase I to II studies from 1996 to 2002 [25]. The analysis showed that response in two-thirds of the patients after haematopoietic stem cell transplantation was durable, with an acceptable transplantrelated mortality of $8.7 \%$. Based on these findings, a prospective, randomized trial is in progress in Europe.

\section{Lessons from observational studies of databases and registries}

Although clinical trials are a valuable means of assessing new treatments, in SSc the lack of a definitive clinical trial template with appropriate end-points for measuring treatment success in this disease makes interpretation and comparison of findings difficult. The use of observational studies of databases and registries may help to elucidate the clinical course of this heterogeneic disease and assist in the identi- fication of factors that may be employed to enrich the patient population, and so increase the likelihood of demonstrating a treatment effect with the available therapeutic options.

\section{Limitations of clinical trials}

Clinical trials are characterized by their ability to answer very specific, straightforward questions in a well defined relatively homogeneous patient population. Although this can provide important answers, it may not be representative of complex real-life scenarios. For example, the patient populations tend to be highly selected, and long-term outcome studies are notoriously difficult to perform and are often limited in duration because of cost. A frequent problem encountered in rare diseases such as SSc is the difficulty in obtaining adequate patient numbers. As a result, compromises are made in terms of study entry criteria and patient management, which can complicate interpretation of the results. Another important aspect to consider in trials of SSc is the extremely slow progression of disease in many patients and the fact that spontaneous improvement is observed in many. This makes it difficult to show benefits of therapy over placebo. As a result, meaningful results are more likely to be obtained by identifying and targeting patients who are most likely to progress, rather than those who remain stable over the course of a clinical trial. The optimal way to achieve this is a matter of much debate.

In addition to these well controlled clinical trials, which are ideal for testing hypotheses but are not representative of all clinical situations, there are alternative ways to examine clinical experience. These include descriptive or observational studies, such as case reports, case series and collections of patients or specimens. Databases and registries that aim to gather data from large groups of patients can provide a wealth of information. A more analytical approach is analysis of cohorts and use of observational studies to generate specific hypotheses, although such observational studies cannot adequately test these hypotheses. These observational studies can be used to address a variety of features, such as associations, risk factors and, to varying degrees, organ outcomes and survival. Unfortunately, the effects of therapy are inevitably confounded by the methodology unless very large cohorts are used; for uncommon diseases (for instance, SSc) such cohorts are not available in a fully developed and highly enriched form, with the possibility of long-term follow up.

Cohort studies do have significant strengths: they can be used to study high-risk patients even before they develop certain manifestations; several outcomes can be studied for each exposure; and statistical modelling can be applied to determine significance in-depth. There are of course limitations to such studies as well: they are time consuming; their management is challenging; missing data are a major problem; and there are often problems resulting from loss to follow up. Moreover, because they are not controlled, cohort studies comprise populations that are inevitably subject to 


\begin{tabular}{|c|c|c|c|c|c|c|}
\hline & \multicolumn{6}{|c|}{ Antibodies } \\
\hline & $\mathrm{ACA}$ & Th/To & U1RNP & U3RNP & Topo & Pol III \\
\hline Patient characteristics & Female & & MCTD & African American & & \\
\hline Disease subtype & Limited & Limited & Limited & Diffuse & Diffuse & Diffuse \\
\hline Joint/skin involvement & $\downarrow$ Joint & $\downarrow$ Joint & $\uparrow$ Joint & & & $\uparrow \uparrow$ Skin \\
\hline Muscle involvement & & & $\uparrow$ & $\uparrow$ & & \\
\hline Calcinosis & $\uparrow$ & & & & & \\
\hline Acroosteolysis & $\uparrow$ & & & & $\uparrow$ & \\
\hline DUs & Yes & & & & Yes & \\
\hline $\mathrm{PAH} \pm \mathrm{PF}$ & PAH & $\mathrm{PAH}$ & PAH & $\mathrm{PAH}+\mathrm{PF}$ & PF & $\downarrow \downarrow P F$ \\
\hline Renal crisis & & & & Yes & Yes & $\uparrow$ \\
\hline
\end{tabular}

ACA, anticentromere antibodies; DU, digital ulcer; MCTD, mixed connective tissue disease; PAH, pulmonary arterial hypertension; PF, pulmonary fibrosis; Pol III, polymerase III; Topo, topoisomerase; U1RNP, U1 ribonuclear protein; U3RNP, U3 ribonuclear protein.

channelling bias, the effects of changing diagnostic criteria and methodology, and the lack of concomitant comparison populations. Despite these limitations, cohort studies can provide valuable insights, and it may be possible to enrich them to make them more meaningful, for example by including rating scales and staging systems, and by using sophisticated analytical methods to evaluate the entire clinical course and outcome of the patient. They can generate solid hypotheses and can serve as strong supporting, or indeed, contradictory evidence for randomized, controlled trials. In fact, observational studies and controlled trials should be considered complementary methodologies, not competing ones. Over recent years a broad base of clinical information has been gained from sources other than clinical trials, and there are a great number of observational studies, databases and registries in many countries.

\section{Findings from observational databases and patient registries}

Features of systemic sclerosis and its clinical course

Autoantibodies are associated with a number of clinical features of SSc. The degree of pulmonary involvement in patients with different autoantibodies varies considerably (Table 1). It is likely that autoantibodies will play an important role as far as subclassification of SSc and its response to therapy are concerned. Interestingly, patients with antipolymerase III antibodies have minimal severe fibrosis; these patients are likely to have very different outcomes to those of patients with both PAH and PF [26].

Identification of different patterns among patients with early diffuse systemic sclerosis

An analysis of skin scores of the Royal Free Scleroderma Database (1985 to 2004) provided interesting insights into the patterns of disease in patients with early diffuse SSc. At the time of the analysis, the database included 1,742 patients with the expected distribution of $33 \%$ with diffuse disease and a $4: 1$ ratio of women to men. Of these patients, data were available from 196 patients with early diffuse disease (patients within 1 year of disease onset) for whom at least 3 years of follow-up data were available. In addition, 29 patients with early diffuse disease who died within 3 years were identified. Latent trajectory modelling was used to evaluate longitudinal data and so estimate and predict unobserved underlying trajectories among these patients. Three groups of patients were identified: a group of patients with very stable severe disease; a group that had intermediate disease that was initially fairly severe but then improved; and a group that started out with fairly mild disease and ended up very mild. These groupings of patients also correlated with clinical manifestations and outcomes, including major organ based complications and death. The outcome that was most strongly associated with skin score was death during follow up [27]. Such information could prove useful for identifying patients with severe disease who are most likely to benefit from treatment, and for stratifying clinical trial populations to increase the likelihood of observing a treatment effect.

The University of Pittsburgh Scleroderma Databank continues to be a valuable source of data from SSc patients. The database was initiated in 1979 and includes data from 2,500 patients, 28,000 patient visits, a mean total observation time of 8 years, 22,000 patient-years of treatment, almost 10,000 skin scores, and 8,000 HAQ assessments. In an analysis of time to development of severe disease in diffuse SSc, a large group of more than 400 patients from the Pittsburgh Scleroderma Databank was assessed over 10 years. Severe 
disease was defined as renal crisis, heart disease (including congestive heart failure, symptomatic arrhythmias, or severe pericardial effusion) and severe PF (but not $\mathrm{PAH}$ ) with an FVC predicted below 55\%. More than half of these patients experienced organ damage within 4 years of the onset of the very first symptom of SSc (Figure 4). Kidney damage appeared to develop most rapidly, with the majority of patients having developed renal crisis within the first 2 years of SSc onset. However, in recent years, with the use of angiotensin-converting enzyme inhibitors, the incidence of death because of renal crisis has decreased significantly, whereas the incidence of death due to PF has increased.

Lung disease as described above has a variable outcome. Ten-year survival is closely associated with the FVC at the time of first evaluation (Figure 5). In patients with an FVC predicted of under $55 \%$, PF is the cause of death or a major contributing factor in almost three-quarters of patients [28]. In this study, at the time of death patients had SSc symptoms for 10 years but pulmonary symptoms for only 5 years. This could suggest that onset of pulmonary disease occurs relatively late in the disease course. However, when serial pulmonary function tests were investigated in this group of patients, most of the volume loss was found to occur during the initial 4-year period when the patients were essentially asymptomatic [28]. Typically, patients are not seen until they are at $65 \%$ of FVC predicted, when little further deterioration is necessary before severe problems develop.

A retrospective analysis was performed to determine whether any of the therapies used in the management of SSc were of potential benefit in patients with ILD [28]. Changes in mean $\%$ FVC predicted and rate of change over the initial 2 years of drug therapy were evaluated in patients with FVC predicted under $70 \% \quad(n=122)$. Therapies included high-dose prednisone and other immunosuppressive drugs (namely methotrexate, chlorambucil, cyclophosphamide and Dpenicillamine). A group of patients who received no drug was also evaluated. FVC was unchanged or reduced in all groups apart from patients receiving cyclophosphamide over this first 2-year time period. The improvement in the cyclophosphamide group was maintained over time, whereas the other patients either stabilized or deteriorated. This observational study was the one that led to the controlled cyclophosphamide trial discussed above [4].

\section{Pulmonary arterial hypertension in patients with systemic sclerosis}

$\mathrm{PAH}$ is a major complication of SSc, and once it develops 1 -year survival is estimated to be $55 \%$ to $68 \%$, as compared with more than $90 \%$ in SSc patients without PAH $[29,30]$. As such, it is important to be able to identify which SSc patients are at greatest risk for developing $\mathrm{PAH}$. A study that looked at risk factors for $\mathrm{PAH}$ in SSc patients found that $\mathrm{PAH}$ patients had disease of longer duration [31]. No differences in FVC between SSc patients with $\mathrm{PAH}$ and those without

\section{Figure 4}

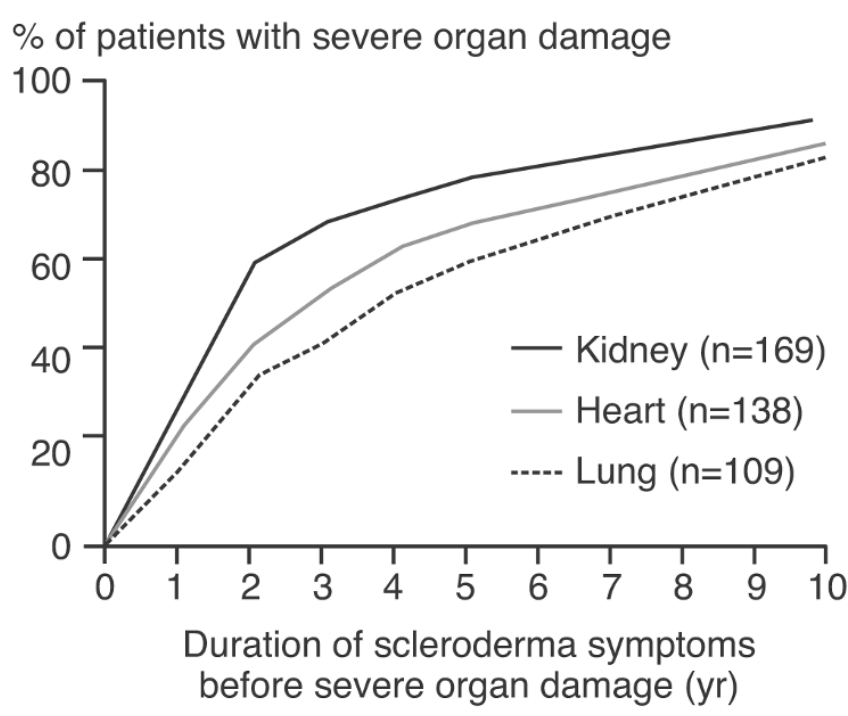

Time to development of severe disease in patients with diffuse SSc. $\mathrm{SSc}$, systemic sclerosis.

\section{Figure 5}

Cumulative survival (\%)

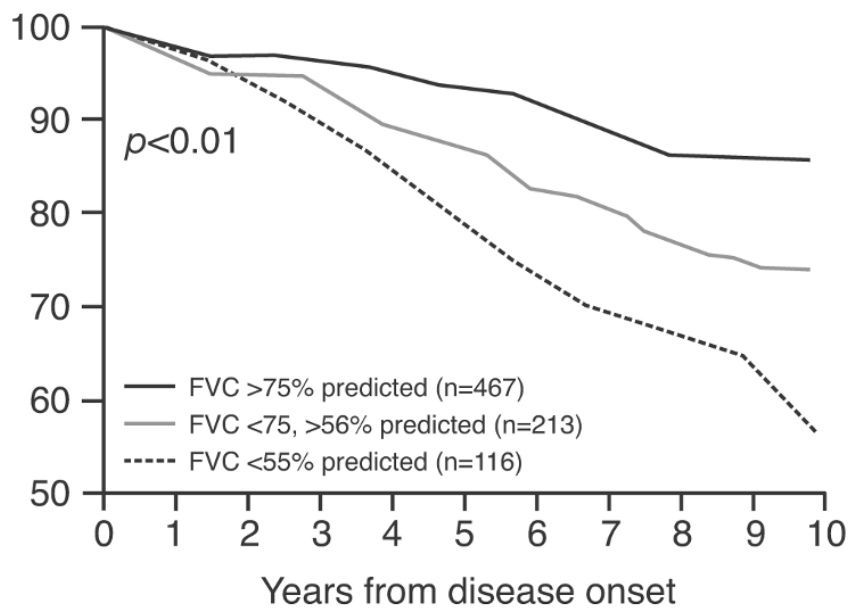

Association between severity of PF and survival in patients with SSc. FVC, forced vital capacity; PF, pulmonary fibrosis; SSc, systemic sclerosis. Reproduced with permission from Steen et al. Arthritis Rheum 1994 (c) John Wiley \& Sons/American College of Rheumatology [28].

$\mathrm{PAH}$ at the time of diagnosis were identified. However, DLco was very low, and more than half of the patients had developed new moderate pericardial effusions. In a casecontrol study, examination of the DLCo 5 years before the echocardiographic diagnosis of $\mathrm{PAH}$ in these patients revealed that even at that time they had a lower mean value compared with control individuals (52\% versus $81 \%)$. 
Additionally, data from a smaller number of patients for whom serial pulmonary function tests were available for up to 15 years before the diagnosis of PAH. These data demonstrated that DLCO decreased over that time from $80 \%$ predicted to $40 \%$ predicted by the time when $\mathrm{PAH}$ was diagnosed, as compared with those who had not developed $\mathrm{PAH}$. In these patients, DLco remained at approximately $80 \%$ predicted.

In the same study, vascular manifestations were also examined in SSc patients with and without PAH [31]. Although patients with and those without $\mathrm{PAH}$ had similar frequencies of Raynaud's (about 96\%) and digital gangrene (8\% to 13\%), the severity of ulcers on the scleroderma HAQ ( 0 to 3 scale) was significantly greater in the $\mathrm{PAH}$ cases than in control individuals $(0.75$ versus $0.45 ; P<0.01)$. In addition, at the time of $\mathrm{PAH}$ diagnosis, a significantly greater proportion of patients had also developed new DUs (38\% versus 10\%; $P<0.05)$. Interestingly, there was significantly lower use of calcium channel blockers in these patients $(P<0.001)$, suggesting that these drugs might have had a preventative effect on development of $\mathrm{PAH}$.

\section{PHAROS}

Existing databases and registries have been able to determine factors in SSc patients that predispose to development of PAH. However, not all patients with a low DLco develop $\mathrm{PAH}$, and it is important to identify those patients who will develop $\mathrm{PAH}$ and over what time period. This is one of the objectives of the Pulmonary Hypertension Assessment Registry of Scleroderma (PHAROS). Other objectives of the registry are to identify the best risk factors for $\mathrm{PAH}$ and to determine the outcomes of patients who develop $\mathrm{PAH}$.

Patients included in the registry are either those deemed to be at high risk for PAH (DLco below 55\% predicted, FVC/DLCO above 1.6 and pulmonary artery systolic pressure on echo above $35 \mathrm{mmHg}$ ) or patients with PAH confirmed by right heart catheterization. The key outcome measures are time to definite $\mathrm{PAH}$ in high-risk patients; in those with definite $\mathrm{PAH}$, the key outcome measures are change in therapy, hospitalization for $\mathrm{PAH}$, and death. Secondary outcomes include 6MWD, pulmonary function testing and dyspnoea questionnaires. It is anticipated that the registry will provide some answers to many of the open questions that remain in the arena of SSc-PAH, which will enable us to initiate prevention trials in enriched populations of patients at high risk for developing $\mathrm{PAH}$ and, ultimately, to improve guidelines for therapy.

\section{Conclusion}

Advances have been made in the treatment of SSc but are hampered by its complex nature and disease heterogeneity. Most nonspecific antifibrotic compounds have exhibited few clinical benefits, although more targeted therapies that address SSc-specific abnormalities are showing some promise. For example, the dual ET-1 receptor antagonist bosentan has yielded positive results in prevention of DUs in SSc patients as well as in the life-threatening complication of $\mathrm{PAH}$ in these patients. Single endothelin receptor antagonists [32] and the phosphodiesterase 5 inhibitor sildenafil [33] are also being evaluated, although not specifically for their effect on DUs.

Results of trials in ILD have highlighted the need for a definitive trial design template for studies in this patient population, and it is becoming increasingly clear that, in a disease such as SSc, the greatest chance of demonstrating a disease-modifying effect is to target the pathogenesis of the disease. Early and aggressive therapy, aimed at the immune system and vasculature, may be best in the early stages of the disease when vascular abnormalities and immunological activity are greatest. Later, when fibrosis is more characteristic and lipase progression slower, antifibrotic therapies not yet developed - may be more appropriate. Key challenges for the future are to develop valid, sensitive, feasible measurement tools and to identify risk factors that will allow the enrichment of randomized, controlled clinical trial patient populations so that clinically relevant treatment effects can be observed.

\section{Competing interests}

JRS has funded research and consultancy relationships with Pfizer Inc, Encysive Pharmaceuticals Inc and Actelion Pharmaceuticals Ltd, which are relevant to this manuscript. VDS has received funds for research lectures, and consulting from Actelion Pharmaceuticals Ltd. DEF and MM-C declare that they have no competing interests.

\section{Acknowledgements}

The authors would like to acknowledge medical writing support funded by an educational grant from Actelion Pharmaceuticals Ltd.

This article is part of Arthritis Research \& Therapy Volume 9 Supplement 2: Advances in systemic sclerosis and related fibrotic and vascular conditions, and is based on presentations made at a symposium entitled Advances in systemic sclerosis and connective tissue disease, sponsored by Actelion Pharmaceuticals Ltd, held in Athens, Greece in April 2006. The full contents of the supplement are available online at http://arthritis-research.com/ supplements/9/S2. This supplement has been supported by an educational grant from Actelion Pharmaceuticals Ltd.

\section{References}

1. Steen VD: Scleroderma renal crisis. Rheum Dis Clin North Am 2003, 29:315-333.

2. Badesch DB, Tapson VF, McGoon MD, Brundage BH, Rubin LJ, Wigley FM, Rich S, Barst RJ, Barrett PS, Kral KM, et al.: Continuous intravenous epoprostenol for pulmonary hypertension due to the scleroderma spectrum of disease. A randomized, controlled trial. Ann Intern Med 2000, 132:425-434.

3. Guillevin L, Gabrielli A, Peter H, Pope J, Morganti A, Denton CP: Long-term effects of bosentan on quality of life, survival, safety and tolerability in pulmonary arterial hypertension associated with connective tissue disease. Ann Rheum Dis 2006, 65(Suppl II):392.

4. Tashkin DP, Elashoff R, Clements PJ, Goldin J, Roth MD, Furst $\mathrm{DE}$, Arriola E, Silver R, Strange C, Bolster M, et al:: Cyclophosphamide versus placebo in scleroderma lung disease. $N$ Engl J Med 2006, 354:2655-2666.

5. Clements PJ, Furst DE, Wong WK, Mayes M, White B, Wigley F, Weisman MH, Barr W, Moreland LW, Medsger TA Jr, et al.: High- 
dose versus low-dose D-penicillamine in early diffuse systemic sclerosis: analysis of a two-year, double-blind, randomized, controlled clinical trial. Arthritis Rheum 1999, 42: 1194-1203.

6. MacLennan $\mathrm{AH}$ : The role of the hormone relaxin in human reproduction and pelvic girdle relaxation. Scand J Rheumatol Supp/ 1991, 88:7-15.

7. Seibold JR, Korn JH, Simms R, Clements PJ, Moreland LW, Mayes MD, Furst DE, Rothfield N, Steen V, Weisman M, et al:: Recombinant human relaxin in the treatment of scleroderma. A randomized, double-blind, placebo-controlled trial. Ann Intern Med 2000, 132:871-879.

8. van den Hoogen FH, Boerbooms AM, Swaak AJ, Rasker JJ, van Lier $\mathrm{HJ}$, van de Putte LB: Comparison of methotrexate with placebo in the treatment of systemic sclerosis: a 24 week randomized double-blind trial, followed by a 24 week observational trial. Br J Rheumatol 1996, 35:364-372.

9. Pope JE, Bellamy N, Seibold JR, Baron M, Ellman M, Carette S, Smith CD, Chalmers IM, Hong P, O'Hanlon D, et al.: A randomized, controlled trial of methotrexate versus placebo in early diffuse scleroderma. Arthritis Rheum 2001, 44:1351-1358.

10. Merkel PA, Silliman NP, Clements PJ, Denton CP, Furst DE, Mayes MD, Pope JE, Polisson RP, Streisand JB, Seibold JR: Patterns and predictors of change in outcome measures in clinical trials in scleroderma. Arthritis Rheum 2005, 52:S282S283.

11. Steen VD, Conte C, Owens GR, Medsger TA Jr: Severe restrictive lung disease in systemic sclerosis. Arthritis Rheum 1994, 37:1283-1289.

12. Abraham D, Distler O: How does endothelial cell injury start? The role of endothelin in systemic sclerosis. Arthritis Res Ther 2007, 9(Suppl 2):S2

13. Krieg $T$, Abraham $D$, Lafyatis R: Fibrosis in connective tissue disease: the role of the myofibroblast and fibroblast-epithelial cell interactions. Arthritis Res Ther 2007, 9(Suppl 2):S4.

14. Denton CP, Merkel PA, Furst DE, Khanna D, Emery P, Hsu VM, Silliman N, Streisand J, Powell J, Akesson A, et al:: Recombinant human anti-TGF $\beta 1$ antibody therapy in systemic sclerosis: a multicenter, randomized, placebo-controlled phase I/II trial of CAT-192. Arthritis Rheum 2007, 56:323-333.

15. Abraham DJ, Vancheeswaran R, Dashwood MR, Rajkumar VS, Pantelides P, Xu SW, du Bois RM, Black CM: Increased levels of endothelin-1 and differential endothelin type $A$ and $B$ receptor expression in scleroderma-associated fibrotic lung disease. Am J Pathol 1997, 151:831-841.

16. Joglekar A, Tsai FS, McCloskey DA, Wilson JE, Seibold JR, Riley DJ: Bosentan in pulmonary arterial hypertension secondary to scleroderma. J Rheumato/ 2006, 33:61-68.

17. Channick RN, Simonneau G, Sitbon O, Robbins IM, Frost A, Tapson VF, Badesch DB, Roux S, Rainisio M, Bodin F, Rubin LJ: Effects of the dual endothelin-receptor antagonist bosentan in patients with pulmonary hypertension: a randomised placebo-controlled study. Lancet 2001, 358:1119-1123.

18. Rubin LJ, Badesch DB, Barst RJ, Galie N, Black CM, Keogh A, Pulido T, Frost A, Roux S, Leconte I, et al.: Bosentan therapy for pulmonary arterial hypertension. N Engl J Med 2002, 346:896903.

19. Seibold JR, Black CM, Denton CP, Furst DE, Guillevin L, Rubin LJ, Wells A, Charef P, Roux S, Chadha-Boreham H: Bosentan versus placebo in interstitial lung disease secondary to systemic sclerosis: the BUILD 2 study [abstract A243]. ATS Poster, May 19-24 2006, San Diego, CA, USA.

20. Buch $M H$, Denton $C P$, Furst $D E$, Guillevin $L$, Rubin $L$, Wells $A U$, Matucci-Cerinic M, Riemekasten G, Emery P, Chada-Boreham H, et al:: Submaximal exercise testing in the assessment of interstitial lung disease secondary to systemic sclerosis: reproducibility and correlations of the six minute walk test. Ann Rheum Dis 2007, 66:169-173.

21. Korn $J H$, Mayes $M$, Matucci-Cerinic M, Rainisio M, Pope J, Hachulla E, Rich E, Carpentier P, Molitor J, Seibold JR, et al.: Digital ulcers in systemic sclerosis: prevention by treatment with bosentan, an oral endothelin receptor antagonist. Arthritis Rheum 2004, 50:3985-3993.

22. Seibold JR, Matucci-Cerinic M, Denton CP, Furst DE, Mayes MD, Kramer F, Morganti A, Korn JH: Bosentan reduces the number of new digital ulcers in patients with systemic sclerosis. Ann Rheum Dis 2006, 65:S90.
23. Witek TJ Jr, Mahler DA: Minimal important difference of the transition dyspnoea index in a multinational clinical trial. Eur Respir J 2003, 21:267-272.

24. Nash RA, McSweeney PA, Crofford LJ, Abidi M, Chen CS, Godwin JD, Gooley TA, Holmberg LA, Henstorf G, Lemaistre CF, et al:: High-dose immunosuppressive therapy and autologous hematopoietic cell transplantation for severe systemic sclerosis: long-term follow-up of the U.S. multicenter pilot study. Blood. Prepublished online April 23, 2007; DOI 10.1182/blood2007-02-072389.

25. Farge D, Passweg J, van Laar JM, Marjanovic Z, Besenthal C Finke J, Peter $\mathrm{HH}$, Breedveld FC, Fibbe WE, Black $\mathrm{C}$, et al:: Autologous stem cell transplantation in the treatment of systemic sclerosis: report from the EBMT/EULAR Registry. Ann Rheum Dis 2004, 63:974-981.

26. Steen VD: Autoantibodies in systemic sclerosis. Semin Arthritis Rheum 2005, 35:35-42

27. Shand LM, Lunt M, Silman AJ, Black CM, Denton CP: Relationship between change in skin sclerosis and outcome in diffuse cutaneous systemic sclerosis: application of a linear latent trajectory model [abstract]. Arthritis Rheum 2004, 51:S1048.

28. Steen VD, Lanz JK Jr, Conte C, Owens GR, Medsger TA Jr: Therapy for severe interstitial lung disease in systemic sclerosis. A retrospective study. Arthritis Rheum 1994, 37:12901296.

29. Kawut SM, Taichman DB, Archer-Chicko CL, Palevsky HI, Kimmel $\mathrm{SE}$ : Hemodynamics and survival in patients with pulmonary arterial hypertension related to systemic sclerosis. Chest 2003, 123:344-350.

30. Stupi AM, Steen VD, Owens GR, Barnes EL, Rodnan GP, Medsger TA Jr: Pulmonary hypertension in the CREST syndrome variant of systemic sclerosis. Arthritis Rheum 1986, 29:515-524.

31. Steen V, Medsger TA Jr: Predictors of isolated pulmonary hypertension in patients with systemic sclerosis and limited cutaneous involvement. Arthritis Rheum 2003, 48:516-522.

32. Barst RJ, Langleben D, Frost A, Horn EM, Oudiz R, Shapiro S, McLaughlin V, Hill N, Tapson VF, Robbins IM, et al.: Sitaxsentan therapy for pulmonary arterial hypertension. Am J Respir Crit Care Med 2004, 169:441-447.

33. Galiè N, Ghofrani HA, Torbicki A, Barst RJ, Rubin LJ, Badesch D, Fleming T, Parpia T, Burgess G, Branzi A, et al.: Sildenafil Use in Pulmonary Arterial Hypertension (SUPER) Study Group. Sildenafil citrate therapy for pulmonary arterial hypertension. N Engl J Med 2005, 353:2148-2157. 